\title{
COVID-19 and SARS-CoV-2 host cell entry mediators: Expression profiling of TMRSS4 in health and disease
}

\author{
PERIKLIS KATOPODIS ${ }^{1,2}$, RACHEL KERSLAKE ${ }^{1,2}$, JULIE DAVIES ${ }^{1}$, HARPAL S. RANDEVA $^{3-5}$, \\ KAMALJIT CHATHA $^{5,6}$, MARCIA HALL ${ }^{1,7}$, DEMETRIOS A. SPANDIDOS ${ }^{8}$, VLADIMIR ANIKIN $^{1,2}$, \\ ANDREAS POLYCHRONIS ${ }^{7}$, JAN L. ROBERTUS ${ }^{9}$, IOANNIS KYROU ${ }^{3-5^{*}}$ and EMMANOUIL KARTERIS ${ }^{1,2^{*}}$ \\ ${ }^{1}$ Department of Life Sciences, College of Health, Medicine and Life Sciences, Brunel University London, \\ Uxbridge UB8 3PH; ${ }^{2}$ Division of Thoracic Surgery, The Royal Brompton and Harefield NHS Foundation Trust, \\ Harefield Hospital, London UB9 6JH; ${ }^{3}$ Warwickshire Institute for the Study of Diabetes, Endocrinology and \\ Metabolism (WISDEM), University Hospitals Coventry and Warwickshire NHS Trust, Coventry CV2 2DX; \\ ${ }^{4}$ Aston Medical Research Institute, Aston Medical School, College of Health and Life Sciences, Aston University, \\ Birmingham B4 7ET; ${ }^{5}$ Warwick Medical School, University of Warwick, Coventry CV4 7AL; ${ }^{6}$ Department of \\ Biochemistry and Immunology, University Hospitals Coventry and Warwickshire NHS Trust, Coventry CV2 2DX; \\ ${ }^{7}$ Mount Vernon Cancer Centre, Middlesex HA6 2RN, UK; ${ }^{8}$ Laboratory of Clinical Virology, Medical School, \\ University of Crete, 71409 Heraklion, Greece; ${ }^{9}$ National Heart and Lung Institute, \\ Imperial College London, London SW3 6LY, UK
}

Received January 25, 2021; Accepted February 22, 2021

DOI: $10.3892 / \mathrm{ijmm} .2021 .4897$

\begin{abstract}
Severe acute respiratory syndrome (SARS) coronavirus-2 (SARS-CoV-2), the causative viral agent for the ongoing COVID-19 pandemic, enters its host cells primarily via the binding of the SARS-CoV-2 spike (S) proteins to the angiotensin-converting enzyme 2 (ACE2). A number of other cell entry mediators have also been identified, including neuropilin-1 (NRP1) and transmembrane protease serine 2 (TMPRSS2). More recently, it has been demonstrated that transmembrane protease serine 4 (TMPRSS4) along with TMPRSS2 activate the SARS-CoV-2 S proteins, and enhance the viral infection of human small intestinal enterocytes. To date, a systematic analysis of TMPRSS4 in health and disease is lacking. In the present study, using in silico tools, the gene expression and genetic alteration of TMPRSS4
\end{abstract}

Correspondence to: Dr Emmanouil Karteris, Department of Life Sciences, College of Health, Medicine and Life Sciences, Brunel University London, Kingston Lane, Uxbridge UB8 3PH, UK

E-mail: emmanouil.karteris@brunel.ac.uk

Dr Ioannis Kyrou, Aston Medical Research Institute, Aston Medical School, College of Health and Life Sciences, Aston University, Aston Street, Birmingham B4 7ET, UK

E-mail: i.kyrou@aston.ac.uk

${ }^{*}$ Contributed equally

Key words: transmembrane protease serine 4, pan-cancer, COVID-19, SARS-CoV-2, tropism were analysed across numerous tumours and compared to controls. The observations were also expanded to the level of the central nervous system (CNS). The findings revealed that TMPRSS4 was overexpressed in 11 types of cancer, including lung adenocarcinoma, lung squamous cell carcinoma, cervical squamous cell carcinoma, thyroid carcinoma, ovarian cancer, cancer of the rectum, pancreatic cancer, colon and stomach adenocarcinoma, uterine carcinosarcoma and uterine corpus endometrial carcinoma, whilst it was significantly downregulated in kidney carcinomas, acute myeloid leukaemia, skin cutaneous melanoma and testicular germ cell tumours. Finally, a high TMPRSS4 expression was documented in the olfactory tubercle, paraolfactory gyrus and frontal operculum, all brain regions which are associated with the sense of smell and taste. Collectively, these data suggest that TMPRSS4 may play a role in COVID-19 symptomatology as another SARS-CoV-2 host cell entry mediator responsible for the tropism of this coronavirus both in the periphery and the CNS.

\section{Introduction}

Severe acute respiratory syndrome (SARS) coronavirus-2 (SARS-CoV-2) is the causative viral agent for the ongoing COVID-19 pandemic, with $>75$ million infected individuals and $>2.38$ million deaths worldwide, up to the writing of the present study (February 14, 2021) (1,2). During the writing of the present manuscript, a glimpse of hope appeared on the horizon with vaccinations being developed and administered globally (3-7).

It is now well-established that the entry of SARS-CoV-2 into cells is facilitated by its spike (S) proteins, mainly through binding to the angiotensin-converting enzyme 2 
(ACE2) $(8,9)$. Moreover, the SARS-CoV-2 S proteins are primed/activated by the transmembrane protease serine 2 (TMPRSS2), which appears to also play a key role in this viral infection $(8,10,11)$. As such, there is increasing interest in identifying additional molecular mediators that may also facilitate the SARS-CoV-2 infection of host cells and promote further adverse COVID-19 symptoms. Accordingly, neuropilin-1 (NRP1) has been identified as a novel cellular mediator implicated in the SARS-CoV-2 infection (12-14), whilst similar evidence has also more recently emerged for TMPRSS4, another transmembrane protease. Indeed, TMPRSS4 - along with TMPRSS2 - appear to activate the SARS-CoV-2 S proteins, and enhance subsequent viral infection of human small intestinal enterocytes (15). These data from Zang et al, provide evidence that the intestine is an additional target organ for SARS-CoV-2 (15). Moreover, a recent study demonstrated that both ACE2 and TMPRSS2 genes were abundantly expressed in enterocytes of the lower gastrointestinal (GI) tract, whilst also exhibiting co-expression with TMPRSS4, particularly in the small intestine (16). Of note, whereas COVID-19 presentation usually includes fever, cough, pulmonary/respiratory symptoms, and loss of taste and smell $(17,18)$, an increasing number of studies has also reported GI-related symptoms (e.g., diarrhoea, nausea and vomiting) in patients with COVID-19 (19-21). Indeed, a proportion of these patients with extra-pulmonary symptoms do not exhibit other respiratory symptoms (22).

Apart from respiratory and GI-related symptoms, it is now evident that patients with COVID-19 may experience a wide repertoire of neurological symptoms and complications (23-28), suggesting that SARS-CoV-2 can also attack the central nervous system (CNS), potentially via additional cell entry mediators, including NRP1 $(14,29)$. Indeed, in silico analysis indicated that certain key mediators which facilitate the entry of SARS-CoV-2 into host cells, including ACE2, Cathepsin L (CTSL), TMPRSS2 and TMPRSS4, are expressed in the CNS, with the two transmembrane proteases being highly expressed in neurons (30).

Finally, increasing evidence suggests that cancer constitutes an additional risk factor for severe COVID-19 infection, with hospitalised patients with COVID-19 (particularly males and those receiving treatment with chemotherapy) exhibiting a poor prognosis and being associated with a high case-fatality rate (31). The authors of the present study, as well as other researchers have documented the differential expression of SARS-CoV-2 infection mediators in malignant states $(11,32)$. TMPRSS2 in particular, was previously found to be significantly upregulated in rectum adenocarcinoma, as well as in prostate, breast and lung cancer (11). Notably, compared to never-smokers, a similar ACE2 and TMPRSS2 expression, but a higher TMPRSS4 expression, has been found in the human bronchial epithelial cells of current smokers when compared to never-smokers (33).

To date, there is a paucity of published data about the expression profile of TMPRSS4 in cancer. Therefore, present study curated pan-cancer data in terms of TMPRSS4 gene expression using in silico approaches. The TMPRSS4 expression profile is also further expanded by providing more evidence regarding the presence of this transmembrane protease in the CNS and GI track.

\section{Data and methods}

Bioinformatics analysis. The expression analysis of TMPRSS4 was validated through the Genotype-Tissue Expression (GTEx, www.gtexportal.org) and GEPIA (gepia.cancer-pku. cn). Information regarding the TCGA cohort pan-cancer data was acquired through cBioPortal (www.cbioportal.org/) and single cell analysis was done by using the Single Cell Portal (singlecell.broadinstitute.org/single_cell). Datasets accessed for pan-cancer analysis were the following: ACC, adrenocortical carcinoma; BLCA, bladder urothelial carcinoma; BRCA, breast invasive carcinoma; CESC, cervical squamous cell carcinoma and endocervical adenocarcinoma; CHOL, cholangiocarcinoma; COAD, colon adenocarcinoma; DLBC, lymphoid neoplasm diffuse large B cell lymphoma; ESCA, oesophageal carcinoma; GBM, glioblastoma multiforme; HNSC, head and neck squamous cell carcinoma; $\mathrm{KICH}$, kidney chromophobe; KIRC, kidney renal clear cell carcinoma; KIRP, kidney renal papillary cell carcinoma; LAML, acute myeloid leukaemia; LGG, brain lower grade glioma; LIHC, liver hepatocellular carcinoma; LUAD, lung adenocarcinoma; LUSC, lung squamous cell carcinoma; MESO, mesothelioma; OV, ovarian serous cystadenocarcinoma; PAAD, pancreatic adenocarcinoma; PCPG, pheochromocytoma and paraganglioma; PRAD, prostate adenocarcinoma; READ, rectum adenocarcinoma; SARC, sarcoma; SKCM, skin cutaneous melanoma; STAD, stomach adenocarcinoma; TGCT, testicular germ cell tumours; THCA, thyroid carcinoma; THYM, thymoma; UCEC, uterine corpus endometrial carcinoma; UCS, uterine carcinosarcoma; and UVM, uveal melanoma. The visualisation of TMPRSS4 expression in the human brain was performed using the Allen brain atlas with 6 human donors, as assessed by microarray and presented as a heatmap (34). These data were acquired from a publicly available source which underwent all appropriate approvals by the Human Investigation Committees and Institutional Ethics Committees of each institute from which samples were obtained.

Statistical analysis. The method used for differential analysis in the present study was one-way ANOVA, using disease state (Tumour or Normal) as variables for calculating differential expression: Gene expression disease state. The expression data are first $\log 2(\mathrm{TPM}+1)$ transformed for differential analysis and the $\log 2 \mathrm{FC}$ is defined as median (Tumor) - median (Normal). Genes with higher $\mid \log 2 \mathrm{FCl}$ values and lower $q$ values than pre-set thresholds are considered differentially expressed genes. For more information please visit: http:/gepia. cancer-pku.cn/help.html.

\section{Results}

Using the TCGA and GTEX datasets, it was demonstrated that, compared to the normal control, TMPRSS4 was upregulated in 11 cancer datasets (i.e., CESC, COAD, LUAD, LUSC, OV, PAAD, READ, STAD, THCA, UCEC and UCS) (Fig. 1), while it was significantly downregulated in 6 datasets (i.e., $\mathrm{KICH}, \mathrm{KIRC}$, KIRP, LAML, SKCM and TGCT), with three of the latter consisting kidney tumours (i.e., KICK, KIRC and KIRP) (Fig. 2). 

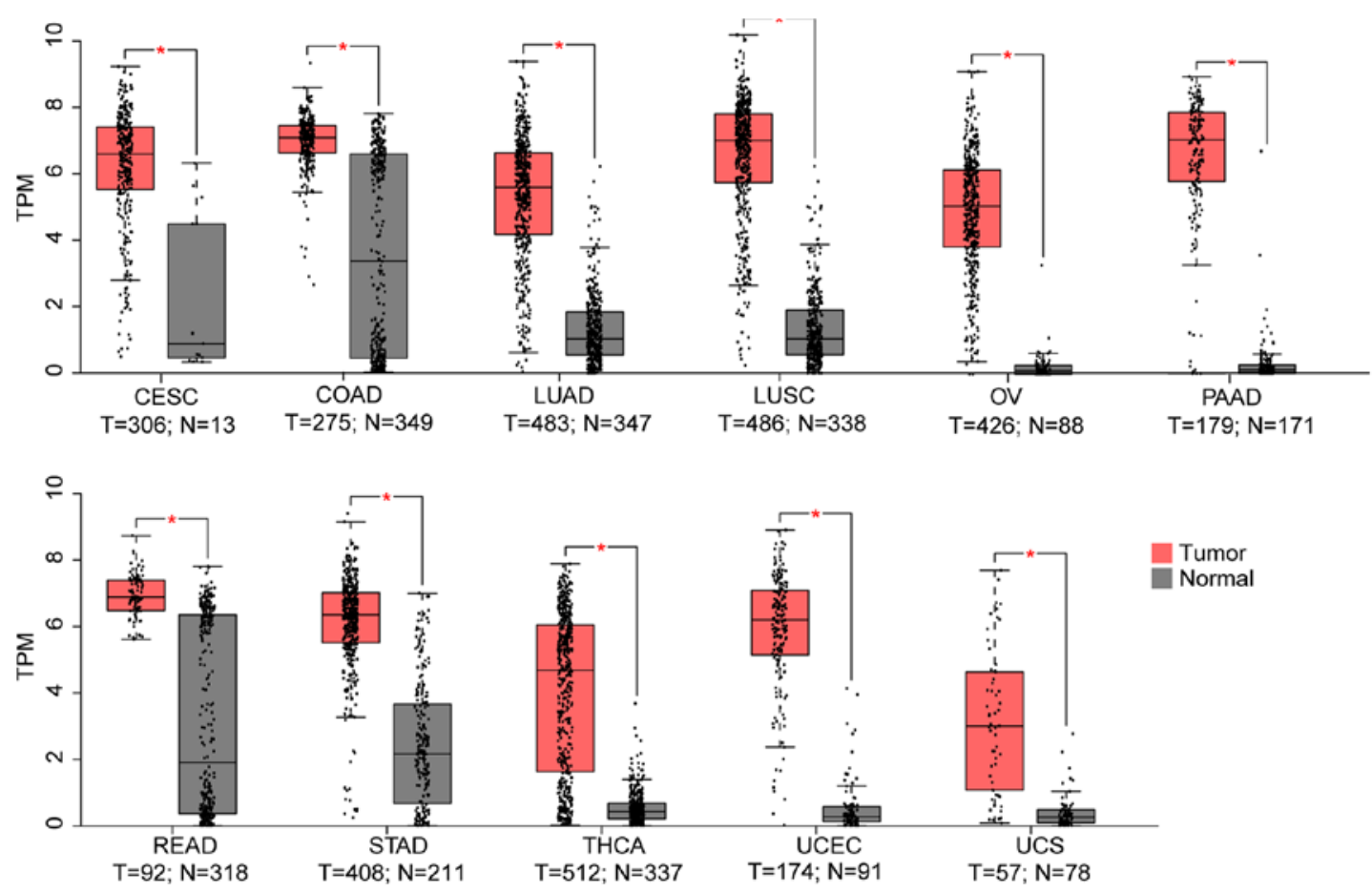

Figure 1. From the 33 TCGA cancer datasets, 11 exhibited a higher TMPRSS4 expression in tumour samples. TMPRSS4 was highly expressed in lung cancers (LUAD and LUSC), gynecological cancers (CESC, OV, UCEC and UCS), pancreatic carcinoma (PAAD), colon adenocarcinoma (COAD), rectum carcinoma (READ), stomach adenocarcinoma (STAD) and thyroid cancer (THCA). T, tumour; N, normal; TPM, transcripts per million; LUAD, lung adenocarcinoma; LUSC, lung squamous cell carcinoma; CESC, cervical squamous cell carcinoma and endocervical adenocarcinoma; OV, ovarian serous cystadenocarcinoma; UCEC, uterine corpus endometrial carcinoma; UCS, uterine carcinosarcoma.

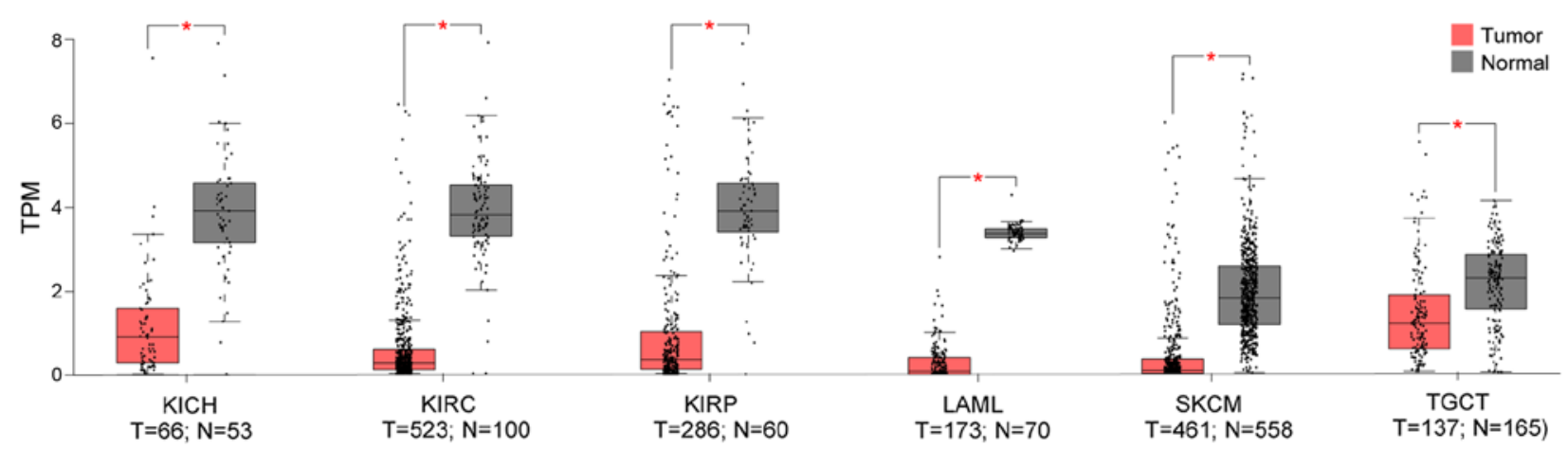

Figure 2. Out of the 33 TCGA cancer datasets, TMPRSS4 was significantly downregulated in 6 datasets (i.e., KICH, KIRC, KIRP, LAML, SKCM and TGCT), with three of these consisting kidney tumours (i.e., KICK, KIRC and KIRP) compared to normal tissues. KICH, kidney chromophobe; KIRC, kidney renal clear cell carcinoma; KIRP, kidney renal papillary cell carcinoma; LAML, acute myeloid leukaemia; SKCM, skin cutaneous melanoma; TGCT, testicular germ cell tumours; T, tumour; N, normal.

Furthermore, using the cBioportal pan-cancer panel, the present study identified specific cancer types with a number of TMPRSS4 amplifications in LGG, LUAD, STAD, deep deletions in BRCA, HNSC, and shallow deletions in BLCA, BRCA, CESC, ESCA, LUAD, LUSC, OV and TGCT. Furthermore, there were two datasets for thyroid cancers with a number of patients presenting diploid and not mutated versions of the gene, namely for THYM and THCA. Of note, in the majority of the studied cancers, the majority of the patients had deletions and partly some gains and amplifications (Fig. 3).

As aforementioned, there is emerging evidence suggesting abundant SARS-CoV-2 infection of the GI tract in severe COVID-19 cases (35). Herein, these observations were expanded upon using single cell analysis from 51 cell subsets
(366,650 cells in total) in the colon mucosa of 18 ulcerative colitis and 12 healthy individuals using the Single Cell Portal (36). Using T-distributed Stochastic Neighbour Embedding (tSNE) - a machine learning algorithm for visualization - distinct cell sub-populations express TMPRSS4 (Fig. 4). Notably, a high TMPRSS4 expression was noted in immature enterocytes, BEST4-expressing enterocytes and enterocytes, as presented in Fig. 4C.

Finally, TMPRSS4 expression was also observed in various brain regions, based on a human microarray data set of 6 human brains (Fig. 5). These data were acquired from a publicly available source which underwent all appropriate approvals by the Human Investigation Committees and Institutional Ethics Committees of each institute from which samples were obtained. 


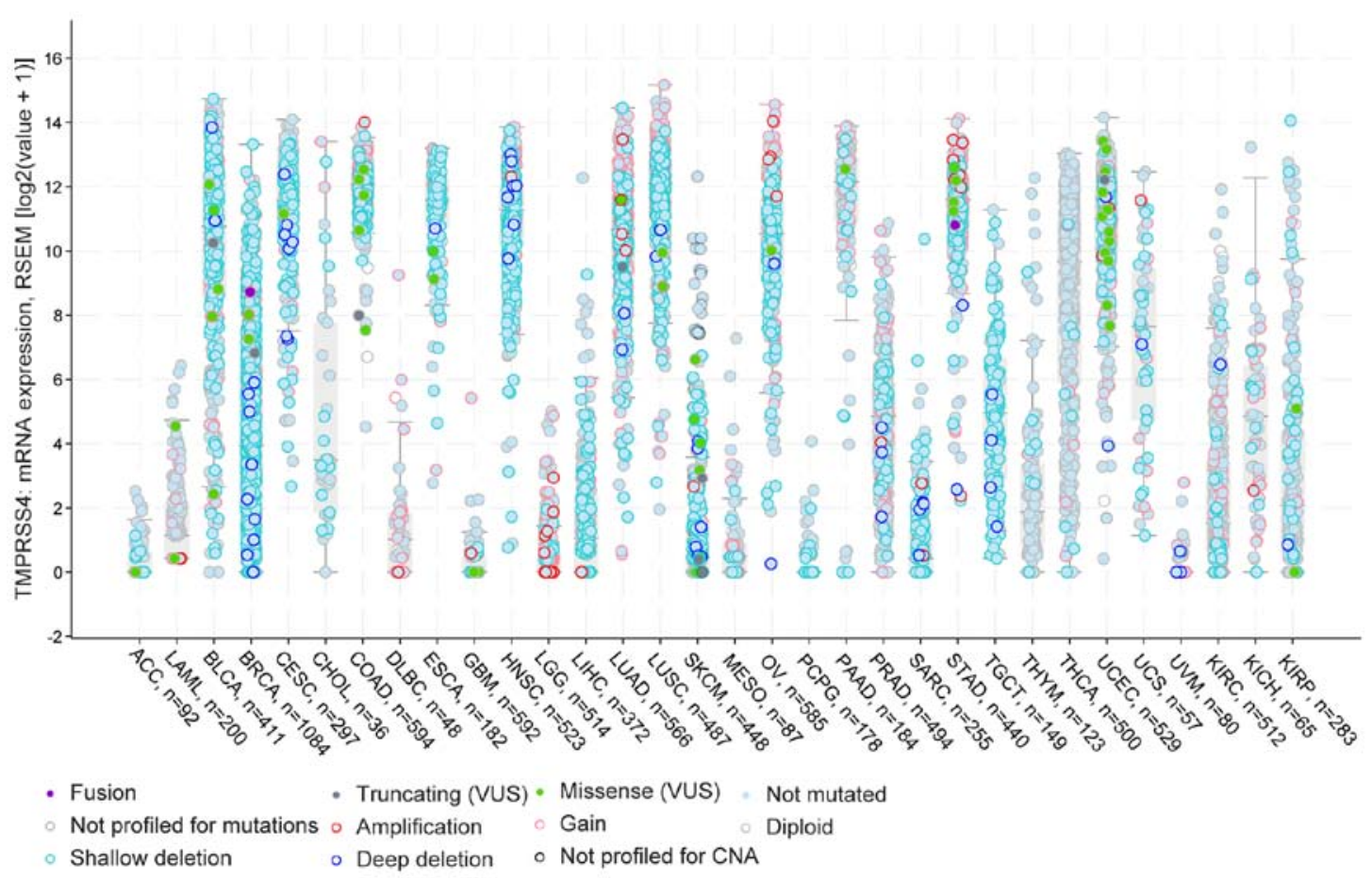

Figure 3. cBioportal analysis of the 32 TCGA datasets with information about the gene dysregulation. In BLCA, LUAD and LUSC there are a number of shallow deletions, while in BRCA and HNSC, high expression and more deep deletions are observed. In gynaecological cancers (CESC, OV, UCEC and UCS), there is high expression of the TMPRSS4 and a few patients present Gains and Missense 'variants of uncertain significant' (VUS). BLCA, bladder urothelial carcinoma; BRCA, breast invasive carcinoma; CESC, cervical squamous cell carcinoma and endocervical adenocarcinoma; HNSC, head and neck squamous cell carcinoma; LUAD, lung adenocarcinoma; LUSC, lung squamous cell carcinoma; OV, ovarian serous cystadenocarcinoma; UCEC, uterine corpus endometrial carcinoma; UCS, uterine carcinosarcoma.

A

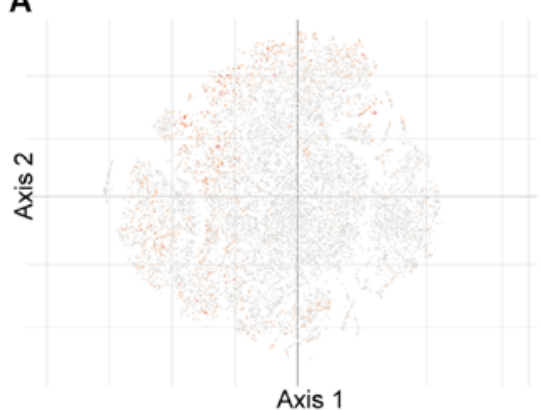

C

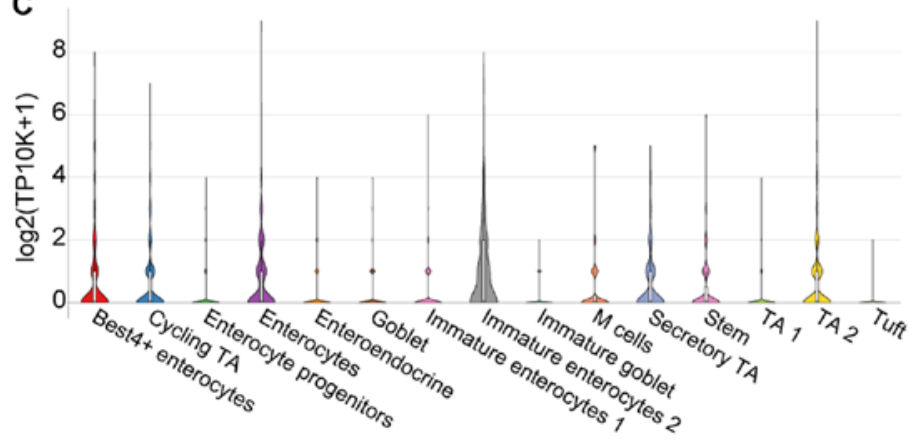

B

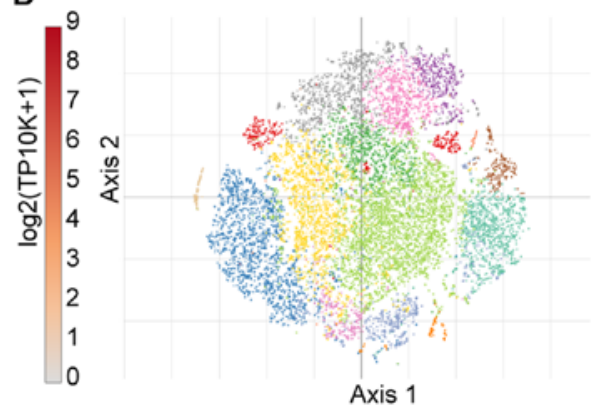

Best4+ enterocytes - Cycling TA Enterocyte progenitors Enterocytes Enteroendocrine Goblet

Immature enterocytes 1 Immature enterocytes 2 Immature goblet $\mathrm{M}$ cells Secretory TA Stem

Figure 4. Single cell analysis of colon mucosa, using the Single Cell Portal, revealed widespread expression of TMPRSS4. (A) Single-cell transcriptomics data are displayed as a spectral tSNE (T-distributed Stochastic Neighbour Embedding) plot of 366,650 cells, annotated according to known cell types; (B) sub-populations of cell types which are enriched for TMPRSS4 expression, with expression intensity demonstrated by a heat map; (C) single cell analysis using the Single Cell Portal, enriched for population subtypes, represented as violin plots. Notably, high TMPRSS4 expression was noted in immature enterocytes, BEST4-expressing enterocytes and enterocytes. TA1, TA2; transit-amplifying (TA) cells.

Fig. 5 presents these data as heatmaps of $\log 2$ expression values, showing that TMPRSS4 is expressed throughout the human brain. Of note, anosmia represents one of the common first symptoms of SARS-CoV-19 infection $(37,38)$, 


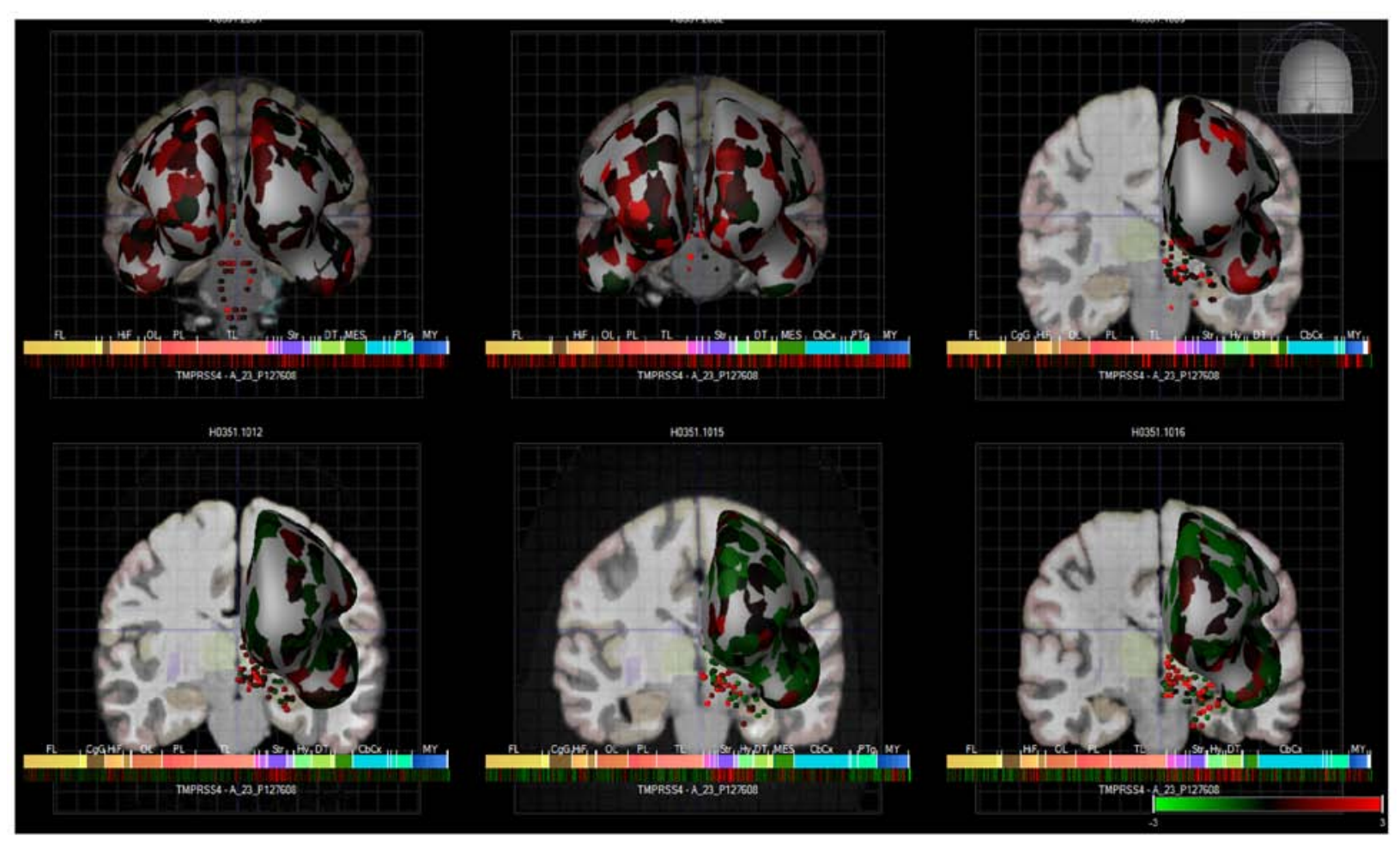

Figure 5. Heatmap expression of TMPRSS4 in the brains of 6 human donors, as assessed by microarray (Allen brain atlas). Global brain expression of TMPRSS4, as detected by microarray probes, with expression of cerebral areas indicated on the hemispheres and expression in brain nuclei indicated as dots. Heatmaps of expression are displayed below individual brains and organised by anterior to posterior regions with the frontal lobe (Fl), hippocampal formation (HiF), occipital lobe $(\mathrm{Ol})$, parietal lobe (Pl), temporal lobe (Tl), striatum (Str), dorsal thalamus (dT), cerebral cortex (cbcx) and myelencephalon (MY) marked on the heatmap.

and although there is global brain expression of TMPRSS4, a high TMPRSS4 expression is noted in brain regions important for the sense of smell and taste, namely in the olfactory tubercle (Log2 expression, 2.1 \pm 1 ), paraolfactory gyrus ( $\log 2$ expression, 1.3 \pm 0.3$)$ and frontal operculum ( $\log 2$ expression, $1.5 \pm 0.5)$. These $\log 2$ expression values are provided by the Allen Brain Atlas. Previous research has also demonstrated that TMPRSS4 exhibited the highest expression in neurons, with the neuronal rich cerebral cortex exhibiting a high TMPRSS4 expression (39). TMPRSS4 protein expression is 1.5 protein-transcripts per million (pTPM) in this region with the highest expression localised to the neuronal cells (ProteinAtlas_ENSG00000137648).

\section{Discussion}

In the present study, comprehensive evidence is presented regarding the peripheral tissue and CNS distribution of a new potential mediator of SARS-CoV-2 infection, namely TMPRSS4. Of note, it was demonstrated that TMPRSS4 is overexpressed in lung cancer; a condition that predisposes to severe COVID-19 $(33,40,41)$. The current findings are in agreement with those of a previous study demonstrating the co-expression of TMPRSS4 with other key SARS-CoV-2 cell entry mediators (i.e., ACE2, ADAM17 and TMPRSS2) in bronchial epithelial cells from never smokers and current smokers (33). Notably, that study also demonstrated that the TMPRSS4 levels were elevated in smokers, suggesting that this may be an additional risk factor for SARS-CoV-2 infection (33). In the lung cancer cohorts investigated in the present study, a marginal difference was note in LUAD with a marked downregulation of TMPRSS4 in current smokers, whilst in patients with LUSC an increase in TMPRSS4 was noted in smokers compared to non-smokers (Fig. S1A and B). This is suggestive of potential tissue-specific effects, given that patients with LUAD have poorer prognosis than those with LUSC (42).

Another recent study also demonstrated a high expression of TMPRSS4 in the human endometrium, which increased with age, particularly in the early phases of the cycle (43). This is of increasing importance given that SARS-CoV-2 infectivity increases with age (44). The present study expanded on these observations and provided evidence of TMPRSS4 expression in other gynaecological tissues, demonstrating that TMPRSS4 is expressed in the cervix, vagina, fallopian tubes, ovaries, breast and uterus, with the first two being the primary organs in terms of a high expression (Fig. S1D). Moreover, it was demonstrated that TMPRSS4 was significantly upregulated in gynaecological malignancies, namely CESC, OV, UCEC and UCS. As age appears to play a role in the risk of COVID-19, the present study further investigated the expression of TMPRSS4 in the above-mentioned malignancies with age. In this respect, TMPRSS4 expression increased with age only in the case of UCEC (Fig. S1C). One of the major limitations of the present study was that in silico data were accessed, that did not allow us to perform statistical analysis with a post hoc test. Another limitation is the absence of survival analysis data. Future studies are required to determine whether TMPRSS 4 can be of prognostic value in terms of overall- and/or progression free survival.

Recently, two different groups have described the involvement of TMPRSS4 as a SARS-CoV-2 entry mediator in the GI $(15,16)$. In particular, ACE2, TMPRSS2 and TMPRSS4 
have been shown to be co-expressed primarily in the small intestine (16). In another study, through a series of elegant experiments, it was shown that, apart from TMPRSS2, TMPRSS4 also enhanced SARS-CoV-2 infectivity in gut epithelial cells (15), suggesting that a leaky gut may allow SARS-CoV-2 to spread to other organs, including the liver. Future studies are required to focus on the role of these key cell entry mediators in GI tract organs, particularly in relation to extra-pulmonary manifestations of severe COVID-19.

Finally, it was demonstrated that TMPRSS4 is present in a number of anterior and posterior regions of the brain. This corroborates previous finding indicating expression in the cerebral cortex, hippocampus and caudate (45). Of note, other SARS-CoV-2 cell entry mediators (e.g., ACE2, TMPRSS2, NRP1 and CTSL) are also expressed in the CNS, raising the possibility of a synergy between multiple such proteins which may further drive the SARS-CoV-2 neurotropism $(29,30,46)$.

In conclusion, the present study documents widespread TMPRSS4 protein expression in the CNS and GI tract, and provides further evidence supporting the potential involvement of TMPRSS4 in facilitating adverse COVID-19 outcomes in patients with certain cancers. Collectively, these data suggest that TMPRSS4 may be implicated in the symptomatology/complications of COVID-19, acting as another SARS-CoV-2 cell entry mediator responsible for the tropism of this novel coronavirus in the periphery and the CNS.

\section{Acknowledgements}

Not applicable.

\section{Funding}

No funding was received.

\section{Availability of data and materials}

All data generated or analysed during this study are included in this published article.

\section{Authors' contributions}

PK, RK, JD, EK, JD, JLR, IK consulted the literature, and produced the figures and manuscript. MH, HSR, KC, JD, VA, DAS, JLR and AP contributed to the literature search, interpretation of the data and the critical revision of the manuscript. PK, JD, HSR, DAS, IK and EK contributed to the writing of the manuscript and final edits. IK and EK contributed equally to the conception of the study, data and literature analysis, as well as interpretation. All authors read and approved the final manuscript. JD is an alumnus of Brunel University London. All authors read and approved the final manuscript.

\section{Ethics approval and consent to participate}

Not applicable.

\section{Patient consent for publication}

Not applicable.

\section{Competing interests}

DAS is the Editor-in-Chief for the journal, but had no personal involvement in the reviewing process, or any influence in terms of adjudicating on the final decision, for this article. The other authors declare that they have no competing interests.

\section{References}

1. World Health Organization (WHO): Weekly Epidemiological Update on COVID-19. WHO, Geneva, 2021.

2. Docea AO, Tsatsakis A, Albulescu D, Cristea O, Zlatian O, Vinceti M, Moschos SA, Tsoukalas D, Goumenou M, Drakoulis N, et al: A new threat from an old enemy: Re-emergence of coronavirus (Review). Int J Mol Med 45: 1631-1643, 2020.

3. Calina D, Hartung T, Docea AO, Spandidos DA, Egorov AM, Shtilman MI, Carvalho F and Tsatsakis A: COVID-19 vaccines: Ethical framework concerning human challenge studies. Daru 28: 807-812, 2020.

4. Kostoff RN, Kanduc D, Porter AL, Shoenfeld Y, Calina D, Briggs MB, Spandidos DA and Tsatsakis A: Vaccine- and natural infection-induced mechanisms that could modulate vaccine safety. Toxicol Rep 7: 1448-1458, 2020.

5. Calina D, Docea AO, Petrakis D, Egorov AM, Ishmukhametov AA, Gabibov AG, Shtilman MI, Kostoff R, CarvalhoF, Vinceti M, et al: Towards effective COVID-19 vaccines: Updates, perspectives and challenges (Review). Int J Mol Med 46: 3-16, 2020.

6. Calina D, Sarkar C, Arsene AL, Salehi B, Docea AO, Mondal M, Islam MT,Zali A and Sharifi-Rad J: Recent advances, approaches and challenges in targeting pathways for potential COVID-19 vaccines development. Immunol Res 68: 315-324, 2020.

7. Voysey M, Clemens SAC, Madhi SA, Weckx LY, Folegatti PM, Aley PK, Angus B, Baillie VL, Barnabas SL, Bhorat QE, et al; Oxford COVID Vaccine Trial Group: Safety and efficacy of the ChAdOx1 nCoV-19 vaccine (AZD1222) against SARS-CoV-2: An interim analysis of four randomised controlled trials in Brazil, South Africa, and the UK. Lancet 397: 99-111, 2021.

8. Hoffmann M, Kleine-Weber H, Schroeder S, Krüger N, Herrler T, Erichsen S, Schiergens TS, Herrler G, Wu NH, Nitsche A, et al: SARS-CoV-2 cell entry depends on ACE2 and TMPRSS2 and is blocked by a clinically proven protease inhibitor. Cell 181: 271-280.e8, 2020.

9. Shang J, Ye G, Shi K, Wan Y, Luo C, Aihara H, Geng Q, Auerbach A and Li F: Structural basis of receptor recognition by SARS-CoV-2. Nature 581: 221-224, 2020.

10. Iwata-Yoshikawa N, Okamura T, Shimizu Y, Hasegawa H, Takeda M and Nagata N: TMPRSS2 contributes to virus spread and immunopathology in the airways of murine models after coronavirus infection. J Virol 93: e01815-e01818, 2019.

11. Katopodis P, Anikin V, Randeva HS, Spandidos DA, Chatha K, Kyrou I and Karteris E: Pan-cancer analysis of transmembrane protease serine 2 and cathepsin $L$ that mediate cellular SARS-CoV-2 infection leading to COVID-19. Int J Oncol 57: 533-539, 2020.

12. Bittmann S, Weissenstein A, Villalon G, Moschuring-Alieva E and Luchter E: Simultaneous treatment of COVID-19 with serine protease inhibitor camostat and/or cathepsin L inhibitor? J Clin Med Res 12: 320-322, 2020.

13. Cantuti-Castelvetri L, Ojha R, Pedro LD, Djannatian M, Franz J, Kuivanen S, Kallio K, Kaya T, Anastasina M, Smura T, et al: Neuropilin-1 facilitates SARS-CoV-2 cell entry and provides a possible pathway into the central nervous system. bioRxiv: doi: https://doi.org/10.1101/2020.06.07.137802.

14. Daly JL, Simonetti B, Klein K, Chen KE, Williamson MK, Antón-Plágaro C, Shoemark DK, Simón-Gracia L, Bauer M, Hollandi R, et al: Neuropilin-1 is a host factor for SARS-CoV-2 infection. Science 370: 861-865, 2020.

15. Zang R, Gomez Castro MF, McCune BT, Zeng Q, Rothlauf PW, Sonnek NM, Liu Z, Brulois KF, Wang X, Greenberg HB, et al: TMPRSS2 and TMPRSS4 promote SARS-CoV-2 infection of human small intestinal enterocytes. Sci Immunol 5: 1-15, 2020.

16. Lee JJ, Kopetz S, Vilar E, Shen JP, Chen K and Maitra A: Relative abundance of SARS-CoV-2 entry genes in the enterocytes of the lower gastrointestinal tract. Genes (Basel) 11: 645, 2020.

17. Guan WJ, Ni ZY, Hu Y, Liang WH, Ou CQ, He JX, Liu L, Shan H, Lei CL, Hui DSC, et al; China Medical Treatment Expert Group for Covid-19: Clinical characteristics of coronavirus disease 2019 in China. N Engl J Med 382: 1708-1720, 2020. 
18. Kerslake R, Hall M, Randeva HS, Spandidos DA, Chatha K, Kyrou I and Karteris E: Co-expression of peripheral olfactory receptors with SARS-CoV-2 infection mediators: Potential implications beyond loss of smell as a COVID-19 symptom. Int J Mol Med 46: 949-956, 2020.

19. Ng SC and Tilg H: COVID-19 and the gastrointestinal tract: More than meets the eye. Gut 69: 973-974, 2020.

20. Almeida JFM and Chehter EZ: COVID-19 and the gastrointestinal tract: What do we already know? Einstein (Sao Paulo) 18 : eRW5909, 2020.

21. Syed A, Khan A, Gosai F, Asif A and Dhillon S: Gastrointestinal pathophysiology of SARS-CoV2 - a literature review. J Community Hosp Intern Med Perspect 10: 523-528, 2020.

22. Jin X, Lian JS, Hu JH, Gao J, Zheng L, Zhang YM, Hao SR, Jia HY, Cai H, Zhang XL, et al: Epidemiological, clinical and virological characteristics of 74 cases of coronavirus-infected disease 2019 (COVID-19) with gastrointestinal symptoms. Gut 69: 1002-1009, 2020.

23. De Felice FG, Tovar-Moll F, Moll J, Munoz DP and Ferreira ST: Severe acute respiratory syndrome coronavirus 2 (SARS-CoV-2) and the central nervous system. Trends Neurosci 43: 355-357, 2020.

24. Mao L, Jin H, Wang M, Hu Y, Chen S, He Q, Chang J, Hong C, Zhou Y, Wang D, et al: Neurologic manifestations of hospitalized patients with coronavirus disease 2019 in Wuhan, China. JAMA Neurol 77: 683-690, 2020.

25. Needham EJ, Chou SH-Y, Coles AJ and Menon DK: Neurological implications of COVID-19 infections. Neurocrit Care 32: $667-671,2020$

26. Mao XY and Jin WL: The COVID-19 pandemic: consideration for brain infection. Neuroscience 437: 130-131, 2020

27. Wu Q, Fan X, Hong H, Gu Y, Liu Z, Fang S, Wang Q, Cai C and Fang J: Comprehensive assessment of side effects in COVID-19 drug pipeline from a network perspective. Food Chem Toxicol 145: 111767,2020

28. Sidiropoulou P, Docea AO, Nikolaou V, Katsarou MS, Spandidos DA, Tsatsakis A, Calina D and Drakoulis N: Unraveling the roles of vitamin D status and melanin during Covid-19 (Review). Int J Mol Med 47: 92-100, 2021.

29. Davies J, Randeva HS, Chatha K, Hall M, Spandidos DA, Karteris E and Kyrou I: Neuropilin-1 as a new potential SARS-CoV-2 infection mediator implicated in the neurologic features and central nervous system involvement of COVID-19. Mol Med Rep 22: 4221-4226, 2020.

30. Matschke J,Lütgehetmann M, Hagel C, Sperhake JP, Schröder AS Edler C, Mushumba H, Fitzek A, Allweiss L, Dandri M, et al: Neuropathology of patients with COVID-19 in Germany: A post-mortem case series. Lancet Neurol 19: 919-929, 2020.

31. Yang K, Sheng Y, Huang C, Jin Y, Xiong N, Jiang K, Lu H, Liu J, Yang J, Dong Y, et al: Clinical characteristics, outcomes, and risk factors for mortality in patients with cancer and COVID-19 in Hubei, China: A multicentre, retrospective, cohort study. Lancet Oncol 21: 904-913, 2020.

32. Chai P, Yu J, Ge S, Jia R and Fan X: Genetic alteration, RNA expression, and DNA methylation profiling of coronavirus disease 2019 (COVID-19) receptor ACE2 in malignancies: A pan-cancer analysis. J Hematol Oncol 13: 43, 2020.

33. Voinsky I and Gurwitz D: Smoking and COVID-19: Similar bronchial ACE2 and TMPRSS2 expression and higher TMPRSS4 expression in current versus never smokers. Drug Dev Res 81: 1073-1080, 2020.
34. Hawrylycz MJ, Lein ES, Guillozet-Bongaarts AL, Shen EH, $\mathrm{Ng}$ L, Miller JA, van de Lagemaat LN, Smith KA, Ebbert A, Riley ZL, et al: An anatomically comprehensive atlas of the adult human brain transcriptome. Nature 489: 391-399, 2012.

35. Ma C, Cong Y and Zhang H: COVID-19 and the digestive system. Am J Gastroenterol 115: 1003-1006, 2020.

36. Smillie CS, Biton M, Ordovas-Montanes J, Sullivan KM, Burgin G, Graham DB, Herbst RH, Rogel N, Slyper M, Waldman J, et al: Intra- and inter-cellular rewiring of the human colon during ulcerative colitis. Cell 178: 714-730.e22, 2019.

37. Walker A, Pottinger G, Scott A and Hopkins C: Anosmia and loss of smell in the era of covid-19. BMJ 370: m2808, 2020

38. Veldhuizen MG and Small DM: Modality-specific neural effects of selective attention to taste and odor. Chem Senses 36: 747-760, 2011.

39. Darmanis S, Sloan SA, Zhang Y, Enge M, Caneda C, Shuer LM, Hayden Gephart MG, Barres BA and Quake SR: A survey of human brain transcriptome diversity at the single cell level. Proc Natl Acad Sci USA 112: 7285-7290, 2015.

40. de Aberasturi AL, Redrado M, Villalba M,Larzabal L, Pajares MJ, Garcia J, Evans SR, Garcia-Ros D, Bodegas ME, Lopez L, et al: TMPRSS4 induces cancer stem cell-like properties in lung cancer cells and correlates with ALDH expression in NSCLC patients. Cancer Lett 370: 165-176, 2016.

41. Larzabal L, Nguewa PA, Pio R, Blanco D, Sanchez B, Rodríguez MJ, Pajares MJ, Catena R, Montuenga LM and Calvo A: Overexpression of TMPRSS4 in non-small cell lung cancer is associated with poor prognosis in patients with squamous histology. Br J Cancer 105: 1608-1614, 2011.

42. Suzuki K, Nagai K, Yoshida J, Nishimura M, Takahashi K, Yokose T and Nishiwaki Y: Conventional clinicopathologic prognostic factors in surgically resected nonsmall cell lung carcinoma. A comparison of prognostic factors for each pathologic TNM stage based on multivariate analyses. Cancer 86: 1976-1984, 1999.

43. Henarejos-Castillo I, Sebastian-Leon P, Devesa-Peiro A, Pellicer A and Diaz-Gimeno P: SARS-CoV-2 infection risk assessment in the endometrium: Viral infection-related gene expression across the menstrual cycle. Fertil Steril 114: 223-232, 2020

44. Nikolich-Zugich J, Knox KS, Rios CT, Natt B, Bhattacharya D and Fain MJ: SARS-CoV-2 and COVID-19 in older adults: What we may expect regarding pathogenesis, immune responses, and outcomes. Geroscience 42: 505-514, 2020. Erratum in: Geroscience 42: 1013, 2020.

45. Guadarrama-Ortiz P, Choreño-Parra JA, Sánchez-Martínez CM, Pacheco-Sánchez FJ, Rodríguez-Nava AI and García-Quintero G: Neurological aspects of SARS-CoV-2 infection: mechanisms and manifestations. Front Neurol 11: 1039, 2020.

46. Frank S: Catch me if you can: SARS-CoV-2 detection in brains of deceased patients with COVID-19. Lancet Neurol 19: 883-884, 2020 .

This work is licensed under a Creative Commons Attribution-NonCommercial-NoDerivatives 4.0 International (CC BY-NC-ND 4.0) License. 